\title{
COROIDEREMIA: SEGUIMIENTO DURANTE UN AÑO CON POLARIMETRÍA LÁSER
}

\section{CHOROIDEREMIA: ONE-YEAR FOLLOW-UP WITH SCANNING LASER POLARIMETRY EXAMINATION}

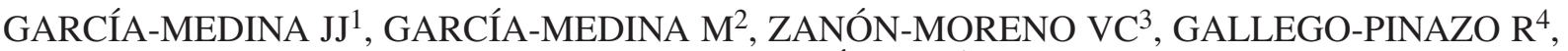 \\ PINAZO-DURÁN MD ${ }^{5}$
}

\section{RESUMEN}

Caso clínico: Presentamos el seguimiento de un caso de coroideremia que fue sometido a tres campos visuales automatizados blanco-blanco y tres polarimetrías láser (PL) con GDx VCC en el transcurso de un año. Se encontró un deterioro perimétrico en índices y escotomas. En concordancia, los parámetros de retardo de la capa de fibras nerviosas de la retina y los mapas cambiaron en un análisis serial avanzado con GDx VCC en los dos ojos.

Discusión: El análisis con GDx VCC puede ser un método objetivo y cuantitativo para evaluar la progresión de las distrofias coriorretinianas como la coroideremia.

Palabras clave: Coroideremia, GDx, progresión, atrofia coriorretiniana, polarimetría láser.

\begin{abstract}
Case report: We report the follow-up of a case of choroideremia who underwent three white-on-white automated visual field and three scanning laser polarimetry (SLP) examinations by means of a GDx VCC in the course of one year. A bilateral perimetric deterioration in indices and scotomas was found. As a result, retinal nerve fiber layer retardation parameters and maps changed on GDx VCC advanced serial analyses in both eyes.

Discussion: Serial analyses with GDx VCC may be used as objective and quantitative tests to assess the progression of chorioretinal dystrophies like choroideremia (Arch Soc Esp Oftalmol 2008; 83: 487492).
\end{abstract}

Key words: Choroideremia, GDx, progression, chorioretinal atrophy, scanning laser polarimetry.

\footnotetext{
Recibido: 5/2/07. Aceptado: 20/6/08.

Unidad de Investigación Oftalmológica Santiago Grisolía. Hospital Universitario Doctor Peset. Valencia. Servicio de Oftalmología. Hospital La Inmaculada. Huercal Overa. Almería. España.

1 Doctor en Medicina.

2 Doctor en Medicina. Servicio de Oftalmología. Hospital Torrecárdenas. Almería.

3 Licenciado en Biología. Unidad de Investigación Oftalmológica Santiago Grisolía. Hospital Universitario Doctor Peset. Valencia.

${ }^{4}$ Licenciado en Medicina. Unidad de Investigación Oftalmológica Santiago Grisolía. Hospital Universitario Doctor Peset. Valencia.

5 Doctora en Medicina. Unidad de Investigación Oftalmológica Santiago Grisolía. Hospital Universitario Doctor Peset. Valencia. Hospital Punta de Europa. Algeciras.
} 


\section{INTRODUCCIÓN}

La coroideremia (CRDM) es una rara distrofia coriorretiniana (DC) de herencia recesiva ligada al cromosoma $\mathrm{X}$ que se caracteriza por una disminución en la visión nocturna y una pérdida progresiva y centrípeta del campo visual periférico.

Los escotomas del campo visual con frecuencia corresponden a áreas de atrofia coriorretiniana (AC) clínicamente discernibles. Con el tiempo, estas áreas coalescen y la constricción del campo visual puede comprobarse mediante perimetría.

Considerando que las $\mathrm{AC}$ aumentan los valores de retardo en la polarimetría láser (PL) realizada con GDx (1), hipotetizamos que la progresión en el tiempo de la CRDM podría ser estimada mediante esta tecnología.

\section{CASO CLÍNICO}

Paciente varón de 53 años previamente diagnosticado de CRDM (nictalopía, constricción progresiva perimétrica, áreas de $\mathrm{AC}$ bien definidas en la angiografía fluoresceínica (fig. 1), electrorretinograma (ERG) alterado (fig. 2), ornitina plasmática normal y patrón de herencia típica ligada a X) y que no tenía otros antecedentes médicos, fue sometido a

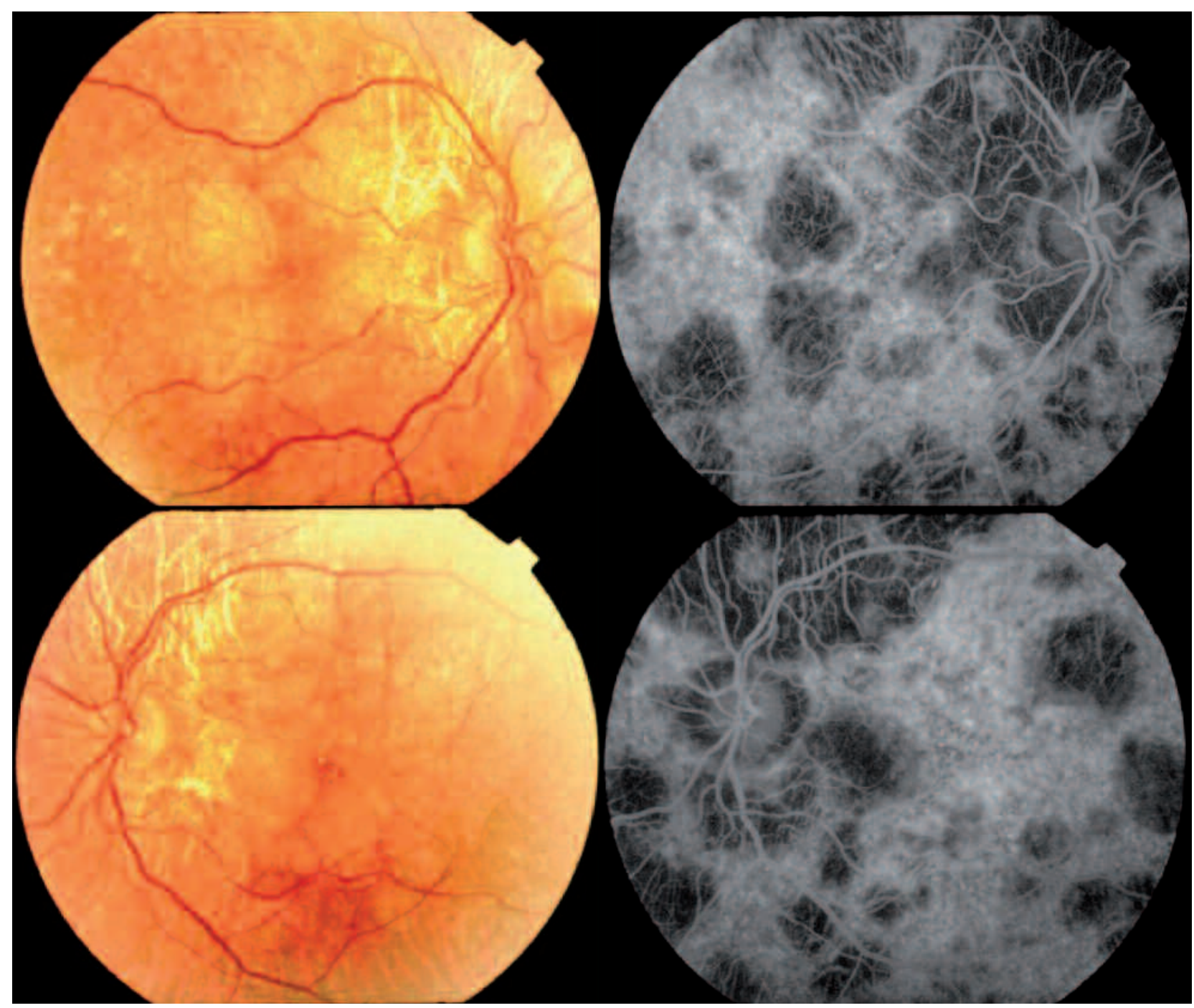

Fig. 1: Funduscopía y angiografía fluoresceínica del ojo derecho (arriba) e izquierdo (abajo). Las áreas parcheadas de atrofia del epitelio pigmentario permiten ver los vasos coroideos infrayacentes. 

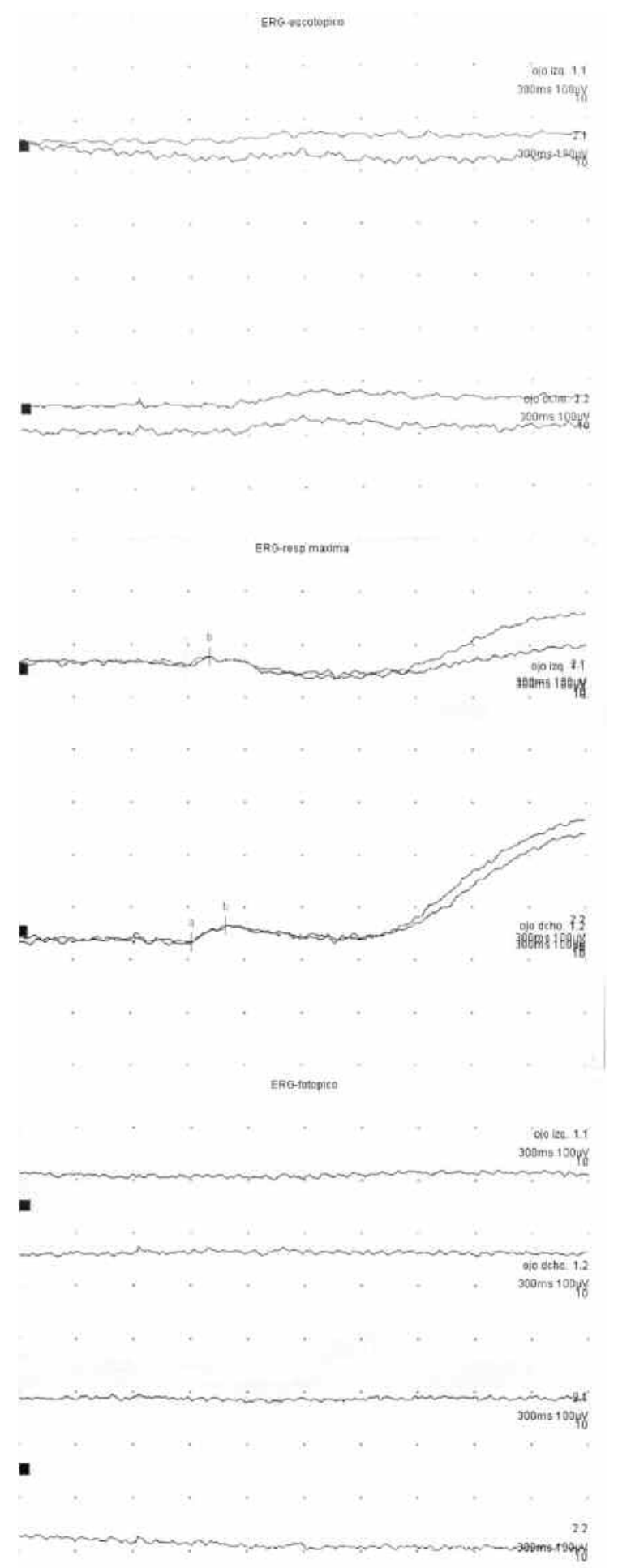

Fig. 2: Electrorretinograma escotópico (arriba), de respuesta máxima (en medio) y fotópico (abajo) de ambos ojos. Se aprecia una afectación retiniana bilateral severa y difusa. tres exámenes oftalmológicos completos por el mismo oftalmólogo en el transcurso de un año que incluyeron agudeza visual mejor corregida (AVMC), campimetrías automatizadas blanco-blanco de los $30^{\circ}$ centrales (Octopus 301, estrategia TOP, programa G1 Haag-Streit, Koeniz, Suiza), polarimetría láser (PL) (GDx with variable corneal compensation (VCC), versión de software 5.5.0; Carl Zeiss Meditec, Dublin, CA, USA), examen con lámpara de hendidura, tonometría de aplanación Goldmann y examen retiniano. Al paciente se le habían realizado 4 campos visuales previos al periodo de seguimiento descrito por lo que se descartó un posible efecto aprendizaje.

La AVMC permaneció en la unidad bilateralmente durante todo el seguimiento. Se observó un deterioro en los escotomas representados en los mapas y en las curvas de Bebie. Además, el defecto medio (MD) empeoró en los exámenes seriados en ambos ojos. Los índices de fiabilidad fueron aceptables. (fig. 3). De forma concordante, se encontraron cambios de forma bilateral en los mapas temporal-superior-nasal-inferior-temporal (TSNIT), los mapas de la capa de fibras nerviosas retinianas (CFNR) y los mapas de la diferencia respecto al estado de base (difference from baseline) en un análisis serial mediante GDx VCC. Además los parámetros absolutos aumentaron y el indicador de fibras nerviosas (NFI) disminuyó. Todos los exámenes con GDx obtuvieron una puntuación de calidad aceptable (Q score mayor o igual a 8) (fig. 3).

Por otro lado, la presión intraocular se mantuvo normal y no se detectaron cambios significativos ni en la biomicroscopía ni en la funduscopía.

\section{DISCUSIÓN}

La medida de los cambios de estado de la polarización de la luz que atraviesa el ojo es una buena técnica para la investigación de los medios oculares. Los dispositivos comerciales desarrollados hasta ahora se centran en el diagnóstico y seguimiento del glaucoma.

Sin embargo, recientemente se ha observado que la PL puede ser útil para otros propósitos clínicos como localizar el punto de fuga y el área de fluido en la coriorretinopatía serosa central (2), comprobar la integridad de las fibras de Henle en la neovascularización secundaria a degeneración macular asociada la edad (3) o cuantificar la opacificación cap- 

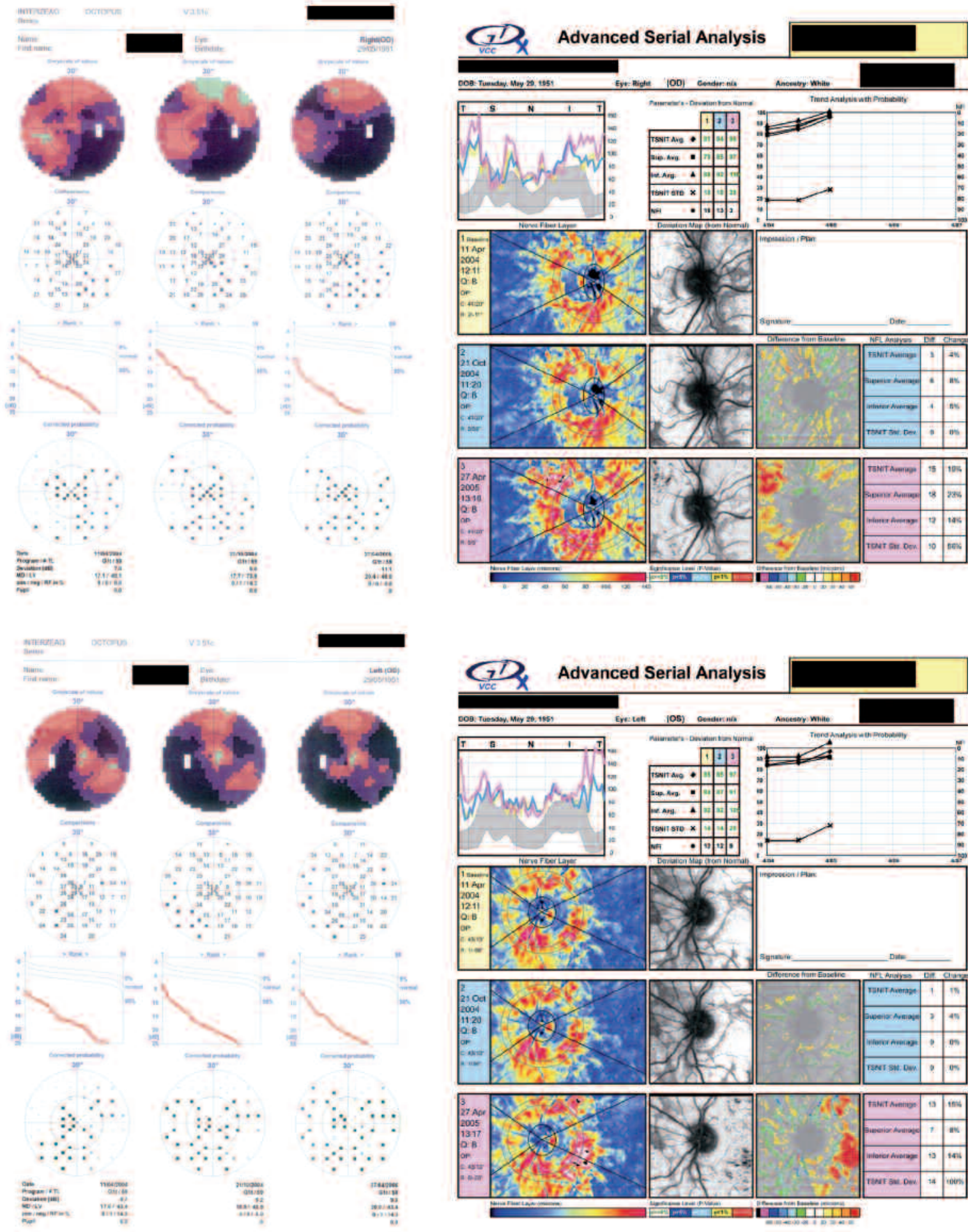

Fig. 3: Perimetría automática y examen GDx VCC del ojo derecho (arriba) y del ojo izquierdo (abajo) del paciente. Nótese como los escotomas se extendieron y/o se hicieron más profundos. Asimismo aparecen nuevos escotomas. El defecto medio (MD) empeoró en ambos ojos. Nótese también la progresión de áreas de aumento de la señal de retardo en los mapas TSNIT, RNFL (retinal nerve fiber layer) y de diferencia respecto al estado de base (difference from baseline). Los parámetros del GDx muestran una tendencia acorde con los mapas en el seguimiento. 
sular posterior en ojos de pacientes no glaucomatosos (4). En nuestro conocimiento éste es el primer reporte en la literatura de la valoración de la progresión de una DC por medio de PL.

El seguimiento fue realizado mediante perimetría automatizada y GDx VCC. La perimetría automatizada es uno de los tests cuantitativos más usados para valorar la progresión de las DC pero sus resultados dependen de la colaboración del paciente y pueden estar alterados por el efecto aprendizaje. Además la perimetría requiere que el paciente preste atención al menos durante varios minutos, incluso en las estrategias más rápidas, así que los niños y los pacientes con enfermedades neuropsiquiátricas no pueden realizar bien este test.

Por otro lado, el GDx VCC es un test objetivo que no depende de la colaboración del paciente. Tarda sólo 0,8 segundos por ojo y se ha demostrado que es una técnica reproducible (5). El único requisito importante es que el paciente pueda mantener la fijación.

Otra prueba estándar usada en el seguimiento de las DC es el ERG. Sin embargo en muchos hospitales el ERG lo realizan los servicios de neurofisiología, con lo que a veces no se puede hacer con la frecuencia deseada. Otra desventaja de esta técnica es la alta variabilidad de resultados entre las visitas.
Aunque se necesitan estudios con un número amplio de pacientes (quizás multicéntricos por la escasez de casos y la lenta evolución de las atrofias), este caso clínico sugiere que la PL mediante GDx VCC puede ser una técnica adecuada para valorar la evolución de las DC progresivas como la CRDM.

\section{BIBLIOGRAFÍA}

1. Bozkurt B, Irkec M, Gedik S, Orhan M, Erdener U, Tatlipinar $S$, et al. Effect of peripapillary chorioretinal atrophy on GDx parameters in patients with degenerative myopia. Clin Experiment Ophthalmol 2002; 30: 411-414.

2. Miura M, Elsner AE, Weber A, Cheney MC, Osako M, Usui $M$, et al. Imaging polarimetry in central serous chorioretinopathy. Am J Ophthalmol 2005; 140: 1014-1019.

3. Weber A, Elsner AE, Miura M, Kompa S, Cheney MC. Relationship between foveal birefringence and visual acuity in neovascular age-related macular degeneration. Eye 2007; 21: 353-361.

4. Garcia-Medina JJ, Garcia-Medina M, Dorta SG, PinazoDuran MD, Gallego-Pinazo R, Zanon-Moreno VC. Effect of posterior capsular opacification removal on scanning laser polarimetry measurements. Graefes Arch Clin Exp Ophthalmol 2006; 244: 1398-1405.

5. Laser Diagnostic Technologies. RNFL analysis with GDxVCC, A primer and clinical guide. San Diego: Laser Diagnostic Technologies; 2004. 